\title{
IMMUNOLOGY
}

\section{Human tumors in immunocompetent mice}

A new platform allows researchers to study the tumor microenvironment in fully immunocompetent chimeric mice.

S ome of the most powerful tools in the study of human antitumor immune responses involve the use of xenotransplantation or transgenic mouse models. However, the study of human tumors in mice poses several challenges, such as weak immune responses or transplant rejection.

Rani George at the Dana-Farber Cancer Institute, Stephani Spranger at the Massachusetts Institute of Technology, Rudolf Jaenisch at the Whitehead Institute for Biomedical Research and colleagues mitigate these issues by developing a mouse-human chimeric model of neuroblastoma, a common childhood malignancy of the peripheral nervous system. To model this disease, the authors generated neural crest cells from human pluripotent stem cells transduced to express oncogenes $M Y C N$ and $A L K$, which were then inserted into mouse embryos.
"Human neuroblastomas developed in immune-competent mice [are] allowing the study of the immune response to human tumors in an in vivo system. This is not possible in any conventional xenotransplantation model as they all rely on immune-compromised hosts," notes co-lead author Rudolf Jaenisch. Herein lies the strength of this study. Having overcome challenges such as manipulation of mouse embryos and construction of human donor cells, the ability to characterize the chimeric immune system is a powerful technological advancement.

Analysis of the tumor microenvironment showed evidence of a significant antitumor immune response that was kept in check by the immunosuppressive tumor milieu. Cytotoxic $\mathrm{CD} 8^{+} \mathrm{T}$ cells from chimeric mice proved to be functional in in vitro cocultures with neuroblastoma cells, confirming a state of T cell exhaustion a loss of function induced by inhibitory stimuli.

The researchers' immediate next step will be to dissect mechanisms of tumor evasion and exploit this knowledge for therapeutic purposes. Thus, in developing an interspecies chimeric tumor model, the researchers present an innovative approach to investigate mechanisms of immune selection pressure and tumor development.

Madhura Mukhopadhyay

Published online: 5 May 2020

https://doi.org/10.1038/s41592-020-0830-z

Research paper

Cohen, M. A. et al. Formation of human

neuroblastoma in mouse-human neural crest chimeras. Cell Stem Cell. 26, 579-592 (2020).

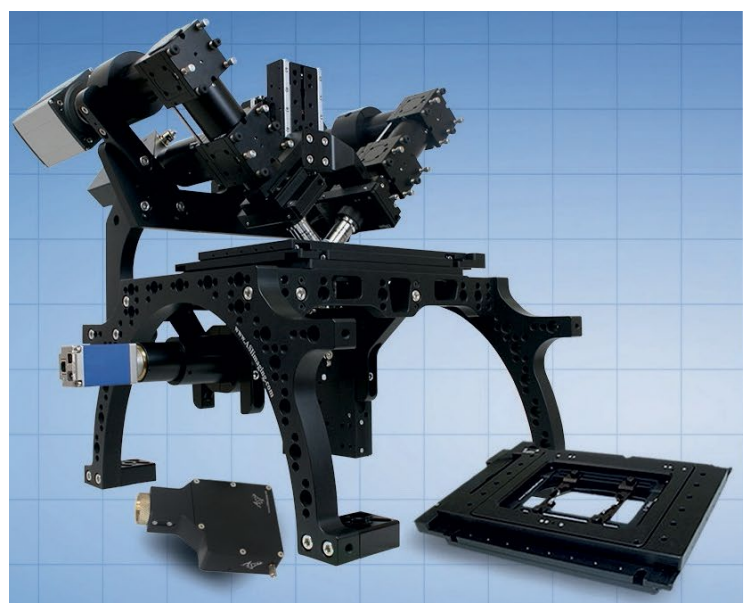



APPLIED SCIENTIFIC INSTRUMENTATION

www.asiimaging.com info@asiimaging.com (800) 706-2284 (541) 461-8181

\section{We Create Solutions}

\author{
Ultra Precise Motion Control - D.C. Servo \\ motors down to $20 \mathrm{~nm}$, piezos down \\ to $1 \mathrm{~nm}$, and low drift XYZ stages.
}

Microscopy - Automation, modular microscopes, autofocus complete light sheet systems, and components.

\section{OEM - Custom designed systems} to user specifications. 